\title{
First results from the Cluster wideband plasma wave investigation
}

\author{
D. A. Gurnett ${ }^{1}$, R. L. Huff ${ }^{1}$, J. S. Pickett ${ }^{1}$, A. M. Persoon ${ }^{1}$, R. L. Mutel ${ }^{1}$, I. W. Christopher ${ }^{1}$, C. A. Kletzing ${ }^{1}$, \\ U. S. Inan ${ }^{2}$, W. L. Martin ${ }^{3}$, J.-L. Bougeret ${ }^{4}$, H. St. C. Alleyne ${ }^{5}$, and K. H. Yearby \\ ${ }^{1}$ Dept. of Physics and Astronomy, University of Iowa, Iowa City, IA 52242, USA \\ ${ }^{2}$ STAR Laboratory, Stanford University, Stanford, CA 94305, USA \\ ${ }^{3}$ Jet Propulsion Laboratory, 4800 Oak Grove Drive, Pasadena, CA 91109, USA \\ ${ }^{4}$ Observatoire de Paris, Place Jules Janssen, 92195 Meudon Cedex, France \\ ${ }^{5}$ University of Sheffield, Automatic Control/Systems Engineering, Sheffield, UK
}

Received: 9 April 2001 - Revised: 15 June 2001 - Accepted: 18 June 2001

\begin{abstract}
In this report we present the first results from the Cluster wideband plasma wave investigation. The four Cluster spacecraft were successfully placed in closely spaced, high-inclination eccentric orbits around the Earth during two separate launches in July - August 2000. Each spacecraft includes a wideband plasma wave instrument designed to provide high-resolution electric and magnetic field waveforms via both stored data and direct downlinks to the NASA Deep Space Network. Results are presented for three commonly occurring magnetospheric plasma wave phenomena: (1) whistlers, (2) chorus, and (3) auroral kilometric radiation. Lightning-generated whistlers are frequently observed when the spacecraft is inside the plasmasphere. Usually the same whistler can be detected by all spacecraft, indicating that the whistler wave packet extends over a spatial dimension at least as large as the separation distances transverse to the magnetic field, which during these observations were a few hundred $\mathrm{km}$. This is what would be expected for nonducted whistler propagation. No case has been found in which a strong whistler was detected at one spacecraft, with no signal at the other spacecraft, which would indicate ducted propagation. Whistler-mode chorus emissions are also observed in the inner region of the magnetosphere. In contrast to lightninggenerated whistlers, the individual chorus elements seldom show a one-to-one correspondence between the spacecraft, indicating that a typical chorus wave packet has dimensions transverse to the magnetic field of only a few hundred $\mathrm{km}$ or less. In one case where a good one-to-one correspondence existed, significant frequency variations were observed between the spacecraft, indicating that the frequency of the wave packet may be evolving as the wave propagates. Auroral kilometric radiation, which is an intense radio emission generated along the auroral field lines, is frequently observed over the polar regions. The frequency-time structure of this radiation usually shows a very good one-to-one correspondence between the various spacecraft. By using the
\end{abstract}

Correspondence to: D. A. Gurnett

(donald-gurnett@uiowa.edu) microsecond timing available at the NASA Deep Space Network, very-long-baseline radio astronomy techniques have been used to determine the source of the auroral kilometric radiation. One event analyzed using this technique shows a very good correspondence between the inferred source location, which is assumed to be at the electron cyclotron frequency, and a bright spot in the aurora along the magnetic field line through the source.

Key words. Ionosphere (wave-particle interactions; wave propagation) - Magnetospheric physics (plasma waves and instabilities; instruments and techniques)

\section{Introduction}

This paper presents the first results from the Cluster wideband plasma wave investigation. The Cluster mission consists of four spacecraft designed to study the fine-scale structure of magnetospheric and solar wind plasma phenomena. The spacecraft were launched on two separate Soyuz-Fregat rockets, the first on 16 July 2000, and the second on $9 \mathrm{Au}-$ gust 2000. After an initial series of maneuvers, the spacecraft were assembled into a closely spaced formation in highinclination eccentric orbits with perigee and apogee geocentric radial distances of about 3.8 and 19.7 Earth radii $\left(R_{E}\right)$. Typical inter-spacecraft separation distances during the early phase of the mission ranged from a few hundred kilometers to about one thousand $\mathrm{km}$, and the orbit period was about 57 hours. A typical orbit is illustrated in Fig. 1, which shows a magnetic meridian plane projection of the trajectory for a two-day period from 27 November to 29 November 2000; the perigee and apogee magnetic local times were about 6.5 and 18.4 hours. Since the orbital planes to first-order remain fixed in inertial space, these local times advance at a rate of 2 hours per month as the Earth revolves around the Sun. For further information on the Cluster spacecraft and the scientific objectives of the Cluster mission, see Escoubet et al. (1997). 


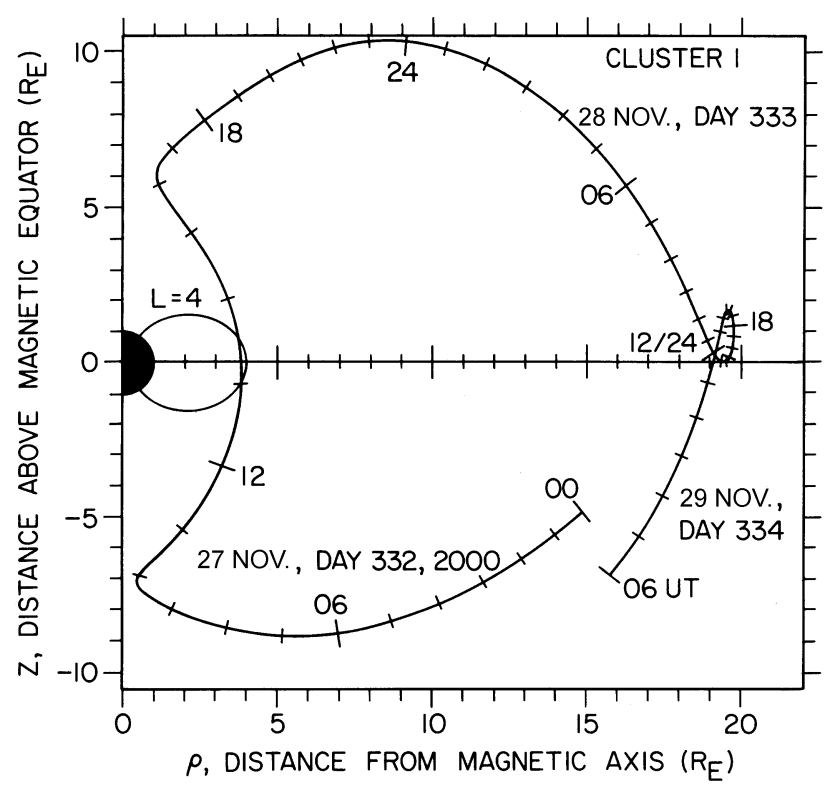

Fig. 1. A typical trajectory of the Cluster spacecraft in magnetic cylindrical coordinates. The coordinate $Z$ is the distance above the magnetic equatorial plane, and the coordinate $\rho$ is the distance from the magnetic axis, both in Earth radii, $R_{E}$. For the orbit shown, which was on 27-29 November 2000, the spacecraft crossed through the magnetic equator near perigee at about 6.5 hours Magnetic Local Time (MLT), and near apogee at about 18.6 hours MLT. For reference, a dipole magnetic field line is shown at $L=4$.

The wideband plasma wave investigation on Cluster is part of the Wave Experiment Consortium (WEC), which consists of five instruments specifically designed to study magnetospheric wave phenomena (Pedersen et al., 1997). The objective of the wideband plasma wave investigation is to provide very high-time resolution measurements in order to resolve the spatial and temporal structure of magnetospheric plasma waves. The investigation consists of four instruments, one on each spacecraft, called the Wideband (WBD) plasma wave instruments. These instruments provide very high-rate digital samples of electric or magnetic field waveforms in three frequency bands, $25 \mathrm{~Hz}$ to $9.5 \mathrm{kHz}, 50 \mathrm{~Hz}$ to $19 \mathrm{kHz}$, and 1 to $77 \mathrm{kHz}$. Waveforms can also be sampled in three frequency conversion modes that provide frequency offsets of 125,250 , and $500 \mathrm{kHz}$. The waveform data can either be recorded onboard the spacecraft for later transmission to the ground, or transmitted real-time to the NASA Deep Space Network (DSN). The bit rate in the real time mode of operation is $220 \mathrm{kbits} \mathrm{s}^{-1}$. Due to limitations of the onboard solid state recorder, full resolution can only be achieved using real time transmission to the Deep Space Network, which is the primary mode of operation. The bandwidths are reduced by about a factor of three when the onboard recorder is used. Real time transmission to the Deep Space Network also allows much better timing accuracy than is available from the onboard spacecraft clock. A timing accuracy of about one microsecond can be achieved via real time transmission to the DSN, versus about one millisecond when using the onboard storage system. Microsecond timing provides the possibility of conducting a number of interesting studies of terrestrial and extraterrestrial radio sources using the wellknown techniques of very-long-baseline radio interferometry. For a description of the Cluster wideband plasma wave investigation, see Gurnett et al. (1997) and for a description of various very-long-baseline-interferometry (VLBI) investigations that can be performed, see Mutel et al. (2000).

Spacecraft post-launch operations were divided into two phases: the commissioning phase, which lasted from launch to 1 February 2001, and the mission operations phase, which started on 1 February 2001. Two types of real time DSN operations were carried out: single-spacecraft and multispacecraft. The single-spacecraft wideband operations are designed primarily to support the WEC by providing highresolution frequency-time spectrograms for the other WEC investigators. The multi-spacecraft wideband operations are intended to use the unique multi-spacecraft configuration of Cluster to resolve the spatial and temporal structure of magnetospheric plasma waves and radio emissions. In this first report, we concentrate on the analysis of data obtained using three or four spacecraft, since these data best utilize the unique multi-point capabilities of Cluster and have the highest potential for producing new results. During the mission operations phase, the commitment of the DSN is to provide 8 hours of single-spacecraft wideband data per orbit, and 1 hour of multi-spacecraft ( 3 or more spacecraft) wideband data once a month. Since only a limited amount of multispacecraft wideband data were obtained during the commissioning phase, and since only a short time has elapsed since the beginning of the mission operations phase, the amount of wideband data available for analysis is quite limited. As of 8 March 2000, a total of seven passes are available for which wideband data were obtained from three or more spacecraft (typically for intervals of about one hour). Five of these intervals were operated in the $25-\mathrm{Hz}$ to $9.5-\mathrm{kHz}$ mode for the purpose of studying whistlers and whistler-mode emissions in the inner region of the magnetosphere near perigee, and two were operated in the frequency-conversion mode for the purpose of studying auroral kilometric radiation at frequencies of 125,250 and $500 \mathrm{kHz}$. Three topics will be discussed using these data: (1) whistlers, (2) chorus, and (3) auroral kilometric radiation.

\section{Whistlers}

Whistlers are very low-frequency electromagnetic waves produced by lightning that have a characteristic whistling sound when converted to audio. These signals were first discovered by Barkhausen (1919) using a rudimentary vacuum tube amplifier, and the relationship to lightning was first suggested by Eckersley (1935). The first comprehensive theory of whistler propagation was developed by Storey (1953), who showed that the signals could be explained if the wave emitted by a lightning stroke propagated through the mag- 
netospheric plasma in a mode of propagation now called the whistler mode (Stix, 1962). Storey showed that at low frequencies, the propagation velocity increases with increasing frequency, thereby causing the high frequency components of the impulsive lightning signal to reach the receiver sooner than the low frequency components, hence the whistling tone. He also showed that the wave energy tends to be guided along the magnetic field such that the wave packet from a lightning stroke in one hemisphere travels approximately along a magnetic field line to the opposite hemisphere where it can be detected as a whistler. Repeated bounces can also occur from one hemisphere to the other along the same magnetic field line. Two types of magnetic guiding are thought to occur: the first is associated with the anisotropy introduced by the Earth's magnetic field, which causes the wave packet to be guided approximately along a magnetic field line; and the second is associated with magnetic fieldaligned density irregularities, which cause the wave packet to be guided almost exactly along the magnetic field, as in a light pipe (Helliwell, 1965). The first type of guiding is called nonducted propagation, and the second type is called ducted propagation. For nonducted propagation, Storey showed that at very low frequencies, the wave energy is confined within a cone that produces an angle of approximately $19^{\circ}$ with respect to the magnetic field. For examples of nonducted ray paths computed using a ray tracing program, see Yabroff (1961). Ducted propagation is more complicated since there is a limited range of wave normal directions that can lead to trapping in a duct; once trapped, the wave is guided almost exactly along the magnetic field line. Ducted propagation accounts for whistlers that are observed to repeatedly bounce back and forth along the same magnetic field line from one hemisphere to the other. For a discussion of the conditions required for ducted propagation, see Smith et al. (1960) and Helliwell (1965). Most whistlers observed on the ground are believed to be caused by ducted propagation, whereas most whistlers observed via spacecraft are believed to be caused by nonducted propagation (Smith and Angerami, 1968; Edgar, 1976). Whistlers observed on the ground are primarily due to ducted propagation because a ducted whistler arrives in the opposite hemisphere with the wave vector nearly vertical, thereby allowing it to be transmitted through the ionosphere to the ground. For nonducted propagation, the whistler arrives in the opposite hemisphere with a very large wave normal angle, thus leading to a magnetospheric reflection, typically before the wave reaches the ionosphere. Such whistlers are called magnetospherically reflected (MR) whistlers (Smith and Angerami, 1968). Whistlers play an important role in radiation belt physics because both ducted (Burgess and Inan, 1993) and nonducted (Johnson et al., 1999) whistlers are known to precipitate energetic electrons via resonant wave-particle interactions. Various modeling studies indicate that whistlers may be important in determining the lifetimes of radiation belt electrons in the inner belt and slot regions (Abel and Thorne, 1998).

Whistlers are frequently observed in the Cluster wideband



Fig. 2. Expanded time scale spectrograms from the four Cluster spacecraft showing the electric field intensities of a whistler detected by all four spacecraft during a perigee pass on 4 February 2001. The frequency scale is in $\mathrm{kHz}$ and the time, labeled UT, is the spacecraft event time in hours:minutes:seconds. The color scale at the top of the illustration gives the electric field spectral density, $E^{2} / \Delta f$, in $\mathrm{Volt}^{2} \mathrm{~m}^{-2} \mathrm{~Hz}^{-1}$, with red representing the most intense and blue representing the least intense. In each case the electric field measurements are from the $Y$-axis electric dipole antenna. The coordinates of all four spacecraft, labeled SC1 through SC4, are given at the bottom of the plot for 13:49:33 UT. $R$ is the geocentric radial distance in $R_{E}$, MLAT is the magnetic latitude in degrees, MLT is the magnetic local time in hours, an $L$ is the McIlwain $L$-shell parameter (McIlwain, 1961).

data. Of the five multi-spacecraft passes through the inner region of the magnetosphere for which real time WBD data were received in the $25-\mathrm{Hz}$ to $9.5-\mathrm{kHz}$ mode, two had whistlers. A total of about two hundred whistlers were detected on these two passes, all located in the region near perigee. An example of a strong whistler detected by Cluster at 13:49:37 Universal Time (UT) on 4 February 2001, is shown in Fig. 2. Unless specifically identified as ground received time, UT time in this paper refers to spacecraft event time. This illustration shows frequency-time spectrograms of the electric field spectral density detected by each of the four spacecraft (labeled SC1 through SC4). The spacecraft at this time were 


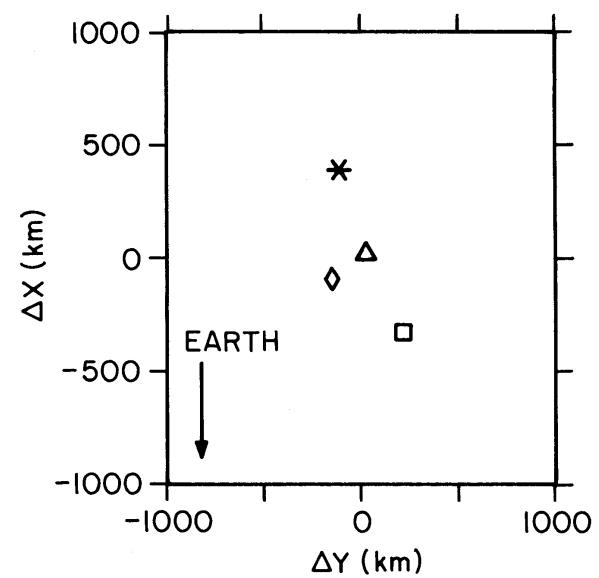

04 - FEB - $2001(035)$

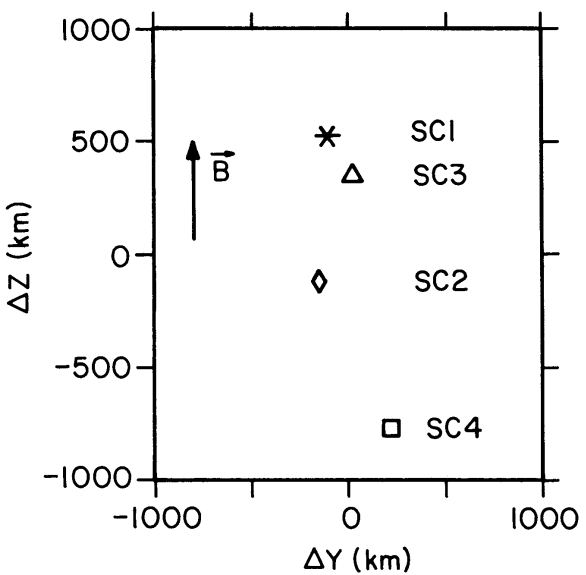

13:49 UT
Fig. 3. The relative geometry of the four Cluster spacecraft in magnetic field-aligned coordinates at the time of the whistler in Fig. 2. The center of the coordinate system is the average position of the four spacecraft (i.e. center of mass), the $\Delta Z$ axis is parallel to the magnetic field $B, \Delta Y$ is in the $\boldsymbol{B} \times \boldsymbol{R}_{\mathrm{cm}}$ direction where $\boldsymbol{R}_{\mathrm{cm}}$ is the radius vector from Earth to the center of mass of the four spacecraft, and the $\Delta X$ axis completes the right-hand coordinate system. The magnetic field direction has been computed using the Tsyganenko model for the Earth's magnetic field (Tsyganenko, 1989). located very close to the magnetic equator at a radial distance of about $4 R_{E}$. The coordinates of the four spacecraft are given at the bottom of the illustration. Since the propagation of whistlers is strongly controlled by the magnetic field direction, the relative positions of the spacecraft in a magnetic field-aligned coordinate system are shown in Fig. 3. The center of this coordinate system is at the center of mass of the four spacecraft, with the $\Delta Z$ axis parallel to the magnetic field $\boldsymbol{B}$. The $\Delta Y$ axis is in the $\boldsymbol{B} \times \boldsymbol{R}_{\mathrm{cm}}$ direction, where $\boldsymbol{R}_{\mathrm{cm}}$ is the radius vector from the Earth to the center of mass of the four spacecraft, and $\Delta X$ completes the righthand system. Note that the spacecraft are strung out in a line, more-or-less along the magnetic field, with SC1 to the north and SC4 to the south.

The whistler in Fig. 2 has a frequency-time shape that closely resembles that of a "nose" whistler, which is a type of whistler often observed on the ground (Carpenter and Smith, 1964). Nose whistlers have a well-defined frequency at which the travel time is a minimum, with the travel time increasing both above and below this frequency (Helliwell, 1965). The increase in the travel time above the frequency of minimum travel time is due to the decrease in the group velocity as the frequency approaches the electron cyclotron frequency. Using magnetic field measurements from the Cluster magnetometers (Balogh et al., 1997), the local electron cyclotron frequency at the time of this whistler was found to range from about $13.5 \mathrm{kHz}$ for spacecraft SC4 to about $14.0 \mathrm{kHz}$ for spacecraft SC3. Since the whistler was detected by all four spacecraft, with separation distances transverse to the magnetic field line of as much as $800 \mathrm{~km}$ (see the $\Delta X-\Delta Y$ panel of Fig. 3), it is clear that this whistler is a nonducted whistler. Typical whistler ducts are thought to have cross-sectional dimensions ranging from 50 to $500 \mathrm{~km}$ (Smith and Angerami, 1968). Although the dispersion properties of nonducted whistlers are somewhat different than ducted whistlers (Edgar, 1976), nose-like features, such as in Fig. 2, occur whenever a nonducted whistler has crossed the magnetic equator.
Careful measurements of the arrival times in Fig. 2 show that the whistler arrived first at SC4 and last at SC1. The difference in the arrival times is about 0.2 seconds. Referring to the $\Delta Z-\Delta Y$ panel of Fig. 3, one can then see that the whistler was propagating from south to north. At first glance, the direction of propagation would appear to indicate a lightning source in the southern hemisphere. However, previous studies of whistlers near the magnetic equator on similar L-shells (Sonwalker and Inan, 1989; Gurnett and Inan, 1988) show that the dispersion is too large for this whistler to have propagated directly to the spacecraft from a source in the southern hemisphere. Instead, we believe that the whistler originated from a lightning source in the northern hemisphere, underwent a magnetospheric reflection at a lower $L$-shell in the southern hemisphere, and was subsequently detected by Cluster on the second (south to north) pass through the magnetic equator. Further examination of panel 4 in Fig. 2 shows that the whistler consists of two components separated by a time delay of about 0.2 seconds. The two closely spaced components are most likely caused by multiple path propagation, although we cannot rule out the occurrence of two closely spaced lightning strokes. The two components look somewhat different at the various spacecraft. For example, at SC2 and SC4, the two components can be clearly resolved, whereas at SC1 and SC3, they cannot be resolved. Clearly the propagation conditions are slightly different over the ray paths to the various spacecraft. Another notable feature is the enhanced background levels that exist for nearly 20 seconds after the whistler has arrived. A poorly defined periodicity can be seen in this diffuse background with a period of about 2 seconds. Such diffuse reflections have been seen before at similar $L$-shells and have been interpreted as being due to subsequent magnetospheric reflections of the original whistler (Sonwalker and Inan, 1989). The diffuse character is believed to be due to the fact that the wave energy enters the magnetosphere over a wide region after propagating long distances in the Earth-ionosphere waveguide. The resulting broad source region at the base of the ionosphere 


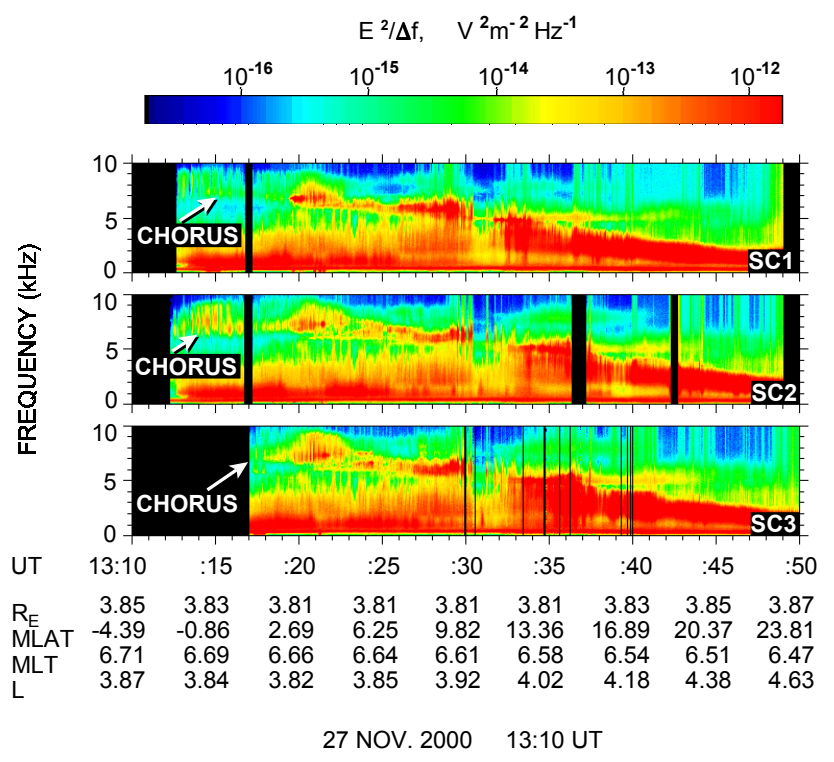

Fig. 4. Frequency-time spectrograms from three of the four Cluster spacecraft during the perigee pass on 27 November 2000. The highly structured emission from about 5 to $8 \mathrm{kHz}$ near the magnetic equator, extending from about 13:12 to 13:32 UT, is chorus. The coordinates given at the bottom of the plot are the average of the coordinates of the three spacecraft (i.e. center of mass).

causes a wide range of propagation paths from the source to the spacecraft, thereby producing a wide range of arrival times. Scattering due to density irregularities in the ionosphere is also believed to be a contributing factor. Scattering spreads the initial wave normal angles, thereby leading to a large range of ray path lengths and travel times. Multiply reflecting whistlers have been proposed as a possible source of plasmaspheric hiss, which is a common feature of the plasmasphere (see Draganov et al., 1992). A steady band of plasmaspheric hiss can be seen at frequencies below about $2 \mathrm{kHz}$ in Fig. 2.

The dispersion characteristics of the whistler in Fig. 2 are typical of essentially all the whistlers detected by Cluster. When the signal is sufficiently strong, the whistler is usually detected at all the spacecraft for which wideband data are available, indicating a predominance of nonducted propagation. We have yet to detect a strong whistler at one spacecraft, with no signal at the other spacecraft, which would be a clear indication of ducted propagation. These observations confirm the conclusion of Smith and Angerami (1968) that ducted whistlers are quite rare.

\section{Chorus}

Chorus, also sometimes called "dawn chorus," is a whistlermode emission that consists of narrowband tones usually rising in frequency on time scales of a few seconds. Storey (1953) describes these signals as sounding like "a rookery heard from a distance," which refers to the sounds made by birds in the morning, hence the term dawn chorus. Early

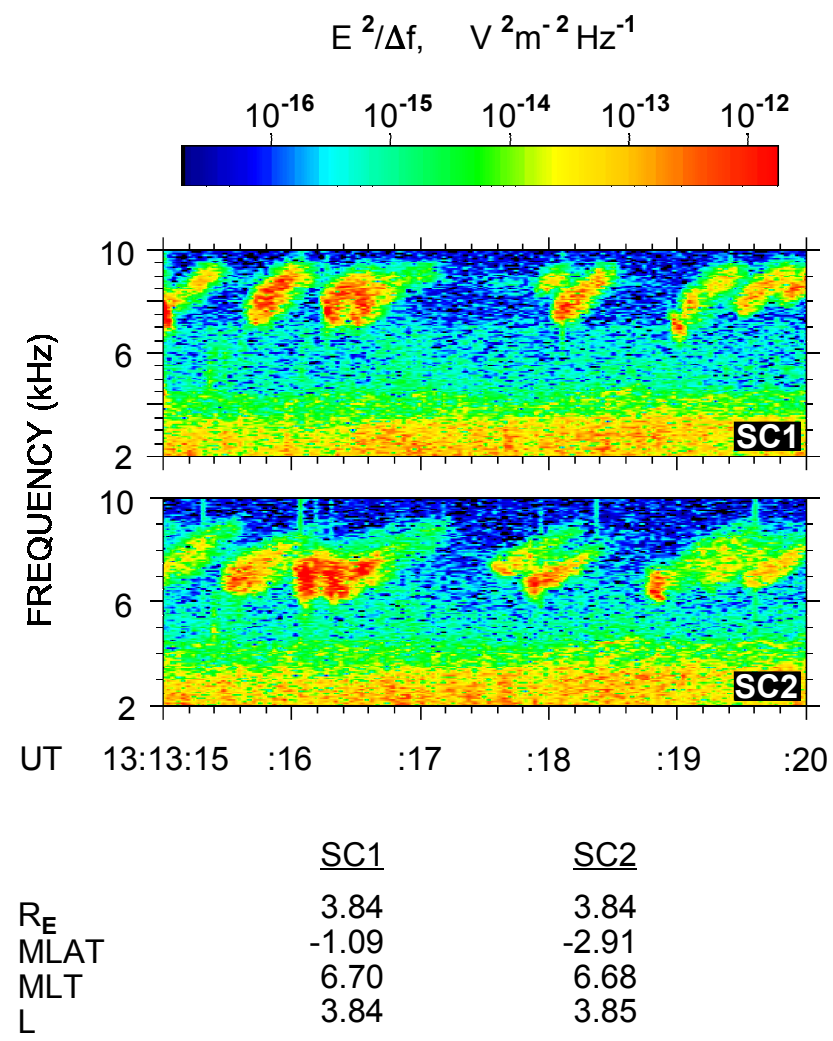

27 NOV. 2000 13:13:15 UT

Fig. 5. Expanded time scale spectrograms of the chorus emissions in Fig. 4 starting at 13:13:15 UT, slightly south of the magnetic equator. The spacecraft coordinates are given at the bottom of the plot for 13:13:15 UT. Note the excellent correlation between the chorus elements.

spacecraft observations showed that chorus occurs over $L$ values ranging from about 3 to 8 , with peak intensities at about $L \approx 5$ (Gurnett and O'Brien, 1964). The intensities of chorus are very high, with wave magnetic field amplitudes from 10 to $100 \mathrm{nT}$, which are among the most intense in the Earth's magnetosphere. It is now known that chorus is generated by trapped radiation belt electrons via a cyclotron resonance mechanism, as first discussed in detail by Kennel and Petschek (1966). Typical resonant energies are on the order of 10 to $100 \mathrm{keV}$ (Burtis and Helliwell, 1975). Chorus thus interacts with ambient energetic electrons, causing pitch-angle scattering and particle precipitation. Chorus emissions are thought to be produced via a loss-cone anisotropy in the energetic ( 10 to $100 \mathrm{keV}$ ) electron distribution within spatial regions which have minimal inhomogeneity in the static magnetic field (Kennel and Petschek, 1966; Burton and Holzer, 1974; Burton, 1976; Sazhin and Hayakawa, 1992, and references therein). Field-aligned focusing of ray paths (by ducts or special features of the refractive index surface) are also thought to play an important role (Burtis and Helliwell, 1975). Early evidence that chorus is generated near the magnetic equator (Helliwell, 1969; Burtis 


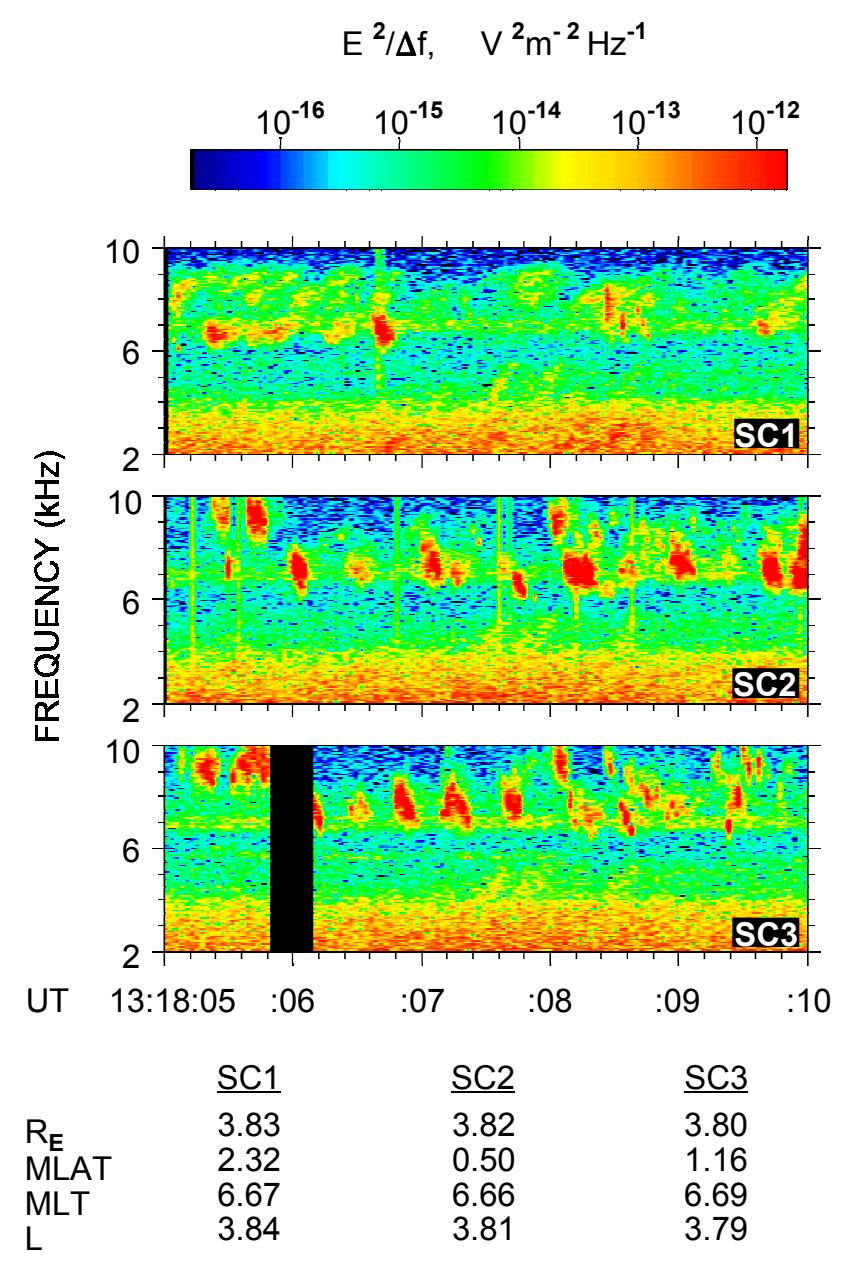

27 NOV. 2000 13:18:05 UT

Fig. 6. Expanded time scale spectrograms of the chorus emissions in Fig. 4 starting at 13:18:05 UT, slightly north of the magnetic equator. The spacecraft coordinates are given at the bottom of the plot for 13:18:05 UT. Note the change in the frequency-time structure compared to Fig. 5, and the poor correlation between the chorus elements at the three spacecraft.

and Helliwell, 1969) has now been confirmed by Poynting flux measurements (LeDocq et al., 1998), which show that the source is located very close to the magnetic equator. The exact reason for the highly coherent discrete frequency-time structure is poorly understood, although the frequency variations are likely to be due to a combination of the inhomogeneous nature of the medium and the nonlinear interaction that arises when resonant electrons are trapped by the magnetic field of the wave (Brice, 1962, 1964; Helliwell, 1967; Nunn et al., 1997). Chorus emissions are thought to drive electron precipitation in the subauroral regions outside the plasmapause and are sometimes directly controlled by the solar wind dynamic pressure (Lauben et al., 1998; Salvati et al., 2000).

Since chorus is known to be generated very close to the magnetic equator, considerable emphasis was placed on ob-

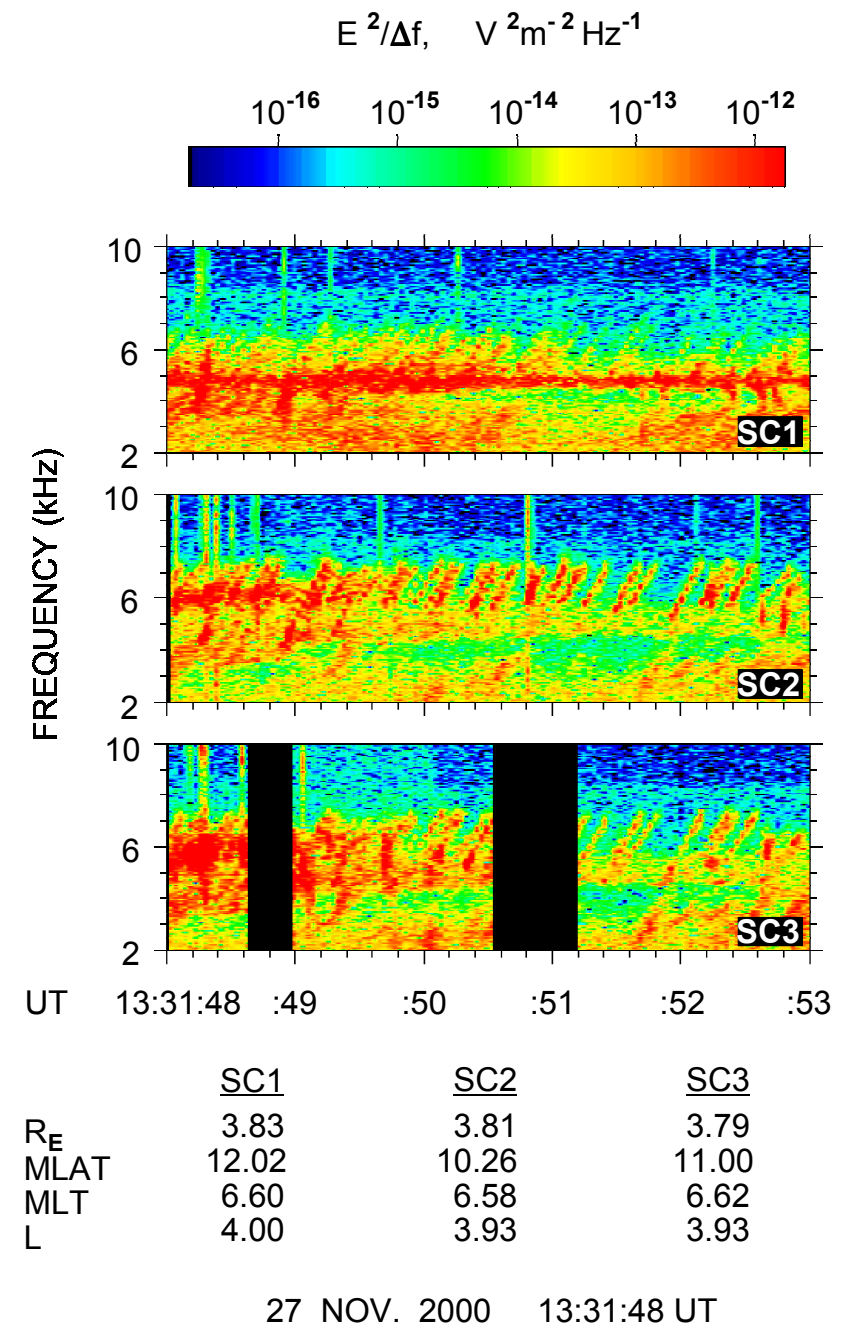

Fig. 7. Expanded time scale spectrogram of the chorus emissions in Fig. 4 starting at 13:31:48 UT, well north of the magnetic equator. The spacecraft coordinates are given at the bottom of the plot for 13:31:48 UT. Again, note the change in the frequency-time structure compared to Fig. 6, and the good correlation between the three spacecraft from about 13:31:50 to 13:31:53 UT.

taining multi-spacecraft wideband observations during perigee passes through the magnetic equator. Of the five passes through this region involving three or more spacecraft in the $25-\mathrm{Hz}$ to $9.5-\mathrm{kHz}$ mode, only one on 27 November 2000, had chorus of sufficient duration and intensity to warrant analysis. This pass occurred in the local morning, at a magnetic local time of about 6.5 hours and a geocentric radial distance of about 3.8 $R_{E}$. The trajectory is shown in Fig. 1 . The paucity of suitable chorus observations is due to the fact that during perigee passes, the spacecraft are often inside the plasmasphere at the magnetic equator. Chorus usually occurs outside the plasmasphere, and it is weak or nonexistent in the plasmasphere. Since the plasmasphere is typically located at $L=4$ to 5 , and moves inward only during periods of enhanced magnetic activity (Carpenter and Anderson, 1992), it was only during magnetically active periods that Cluster had 

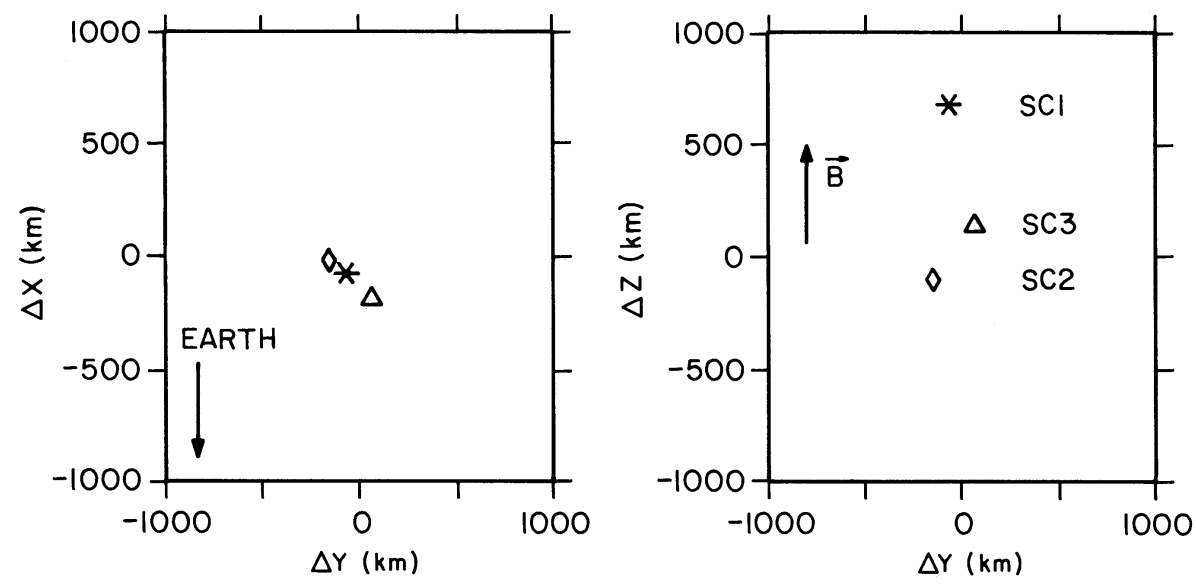

20 - NOV - 2000 (332) 13:13 UT

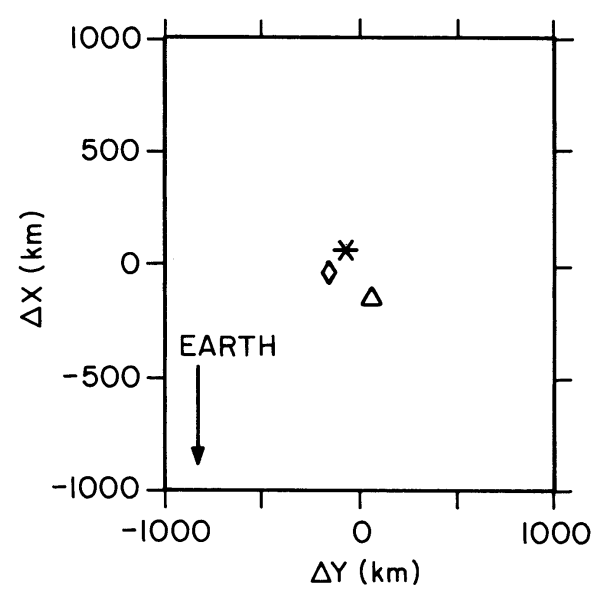

27 - NOV - 2000 (332) 13:19 UT

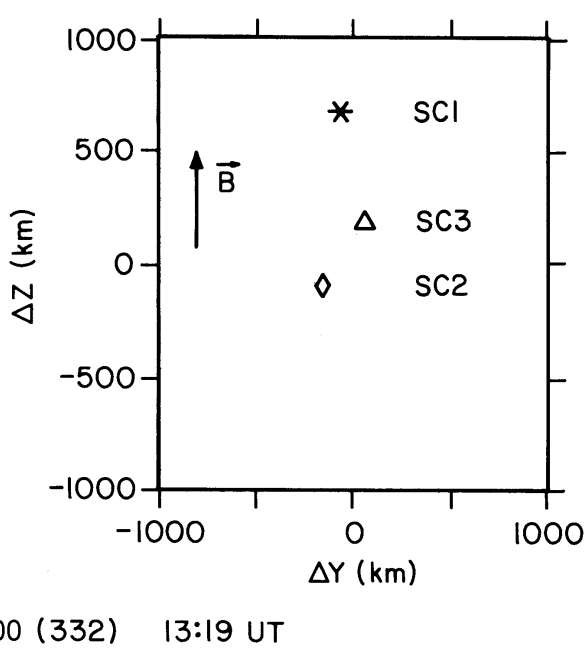

Fig. 8. The relative locations of spacecraft SC1, SC2 and SC3 in magnetic field-aligned coordinates at 13:13 UT on 27 November 2000, using the Tsyganenko model for the Earth's magnetic field (Tsyganenko, 1989). At this time, the spacecraft were slightly south of the magnetic equator. Note the small separation of the three spacecraft in the $\Delta X-\Delta Y$ plane, transverse to the magnetic field.
Fig. 9. The relative locations of spacecraft $\mathrm{SC} 1, \mathrm{SC} 2$ and $\mathrm{SC} 3$ in magnetic field-aligned coordinates at 13:19 UT on 27 November 2000, using the Tsyganenko model for the Earth's magnetic field (Tsyganenko, 1989). At this time, the spacecraft were slightly north of the magnetic equator. Note that the relative separation in the $\Delta X-\Delta Y$ plane, transverse to the magnetic field, is comparable to Fig. 8 . a good chance of detecting chorus. The 27 November pass is one such case. The global magnetic disturbance index, $K p$, reached a peak of $6+$ a few hours before this event and had a value of 4 at the time of the magnetic equator crossing. The lack of chorus events during other later perigee passes could also be due to the fact that after 27 November, the local time was gradually shifting into the local midnight and local evening regions where chorus is much less common.

During the 27 November pass, real time WBD data were received for 38 minutes, from 13:12 UT to 13:50 UT. Frequency-time spectrograms of the electric field spectral densities obtained during this time interval are shown in Fig. 4. The spacecraft coordinates given at the bottom of the spectrogram are averages of the coordinates of the three spacecraft (i.e. center of mass position). The magnetic equator crossing for the three spacecraft combination occurs at 13:16:54 UT. Strong chorus emissions with well-defined discrete structure are observed at frequencies from about 5 to $8 \mathrm{kHz}$ during the entire interval around the magnetic equator crossing. These emissions are marked as "chorus" in Fig. 4.
Hiss-like emissions, similar to what is commonly called plasmaspheric hiss, are observed during the entire pass at frequencies below about $4 \mathrm{kHz}$. Since plasmaspheric hiss usually occurs inside the plasmasphere, the presence of these hiss-like emissions suggests that the spacecraft was probably very close to, but just outside, the plasmapause.

Electric field spectrograms showing the detailed frequency-time structure of the chorus emissions on a greatly expanded (5 second) time scale are given in Figs. 5, 6, and 7. These spectrograms start at 13:13:15 UT, slightly south of the magnetic equator, at 13:18:05 UT, just north of the magnetic equator, and at 13:31:48 UT, well north of the equator. The corresponding spacecraft locations in magnetic field-aligned coordinates are shown in Figs. 8, 9 and 10. The $L$ value over this interval remained nearly constant, varying only from 3.80 to 3.92 . Using the onboard magnetic field measurements, the local electron cyclotron frequency varied from a minimum of $12.2 \mathrm{kHz}$ for SC4 at 13:13:15 UT, to a maximum of $13.9 \mathrm{kHz}$ for SC3 at 13:31:48 UT.

Unfortunately, near the beginning of the pass, no data were 

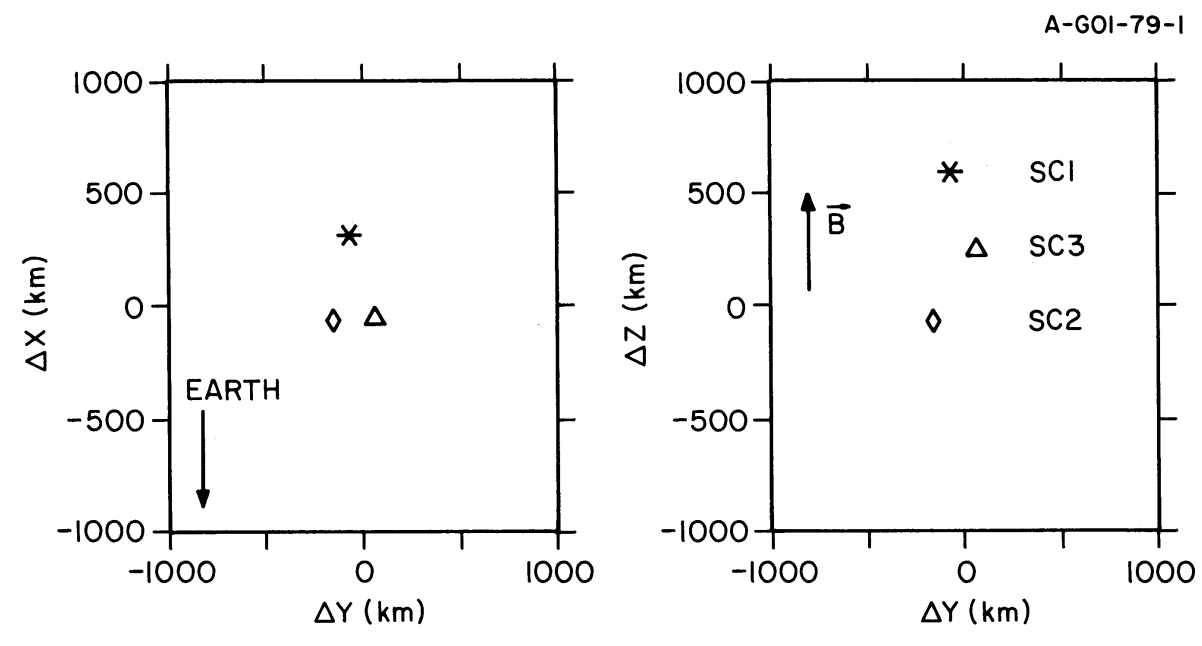

2000-NOV-27 (332) 13:31 UT

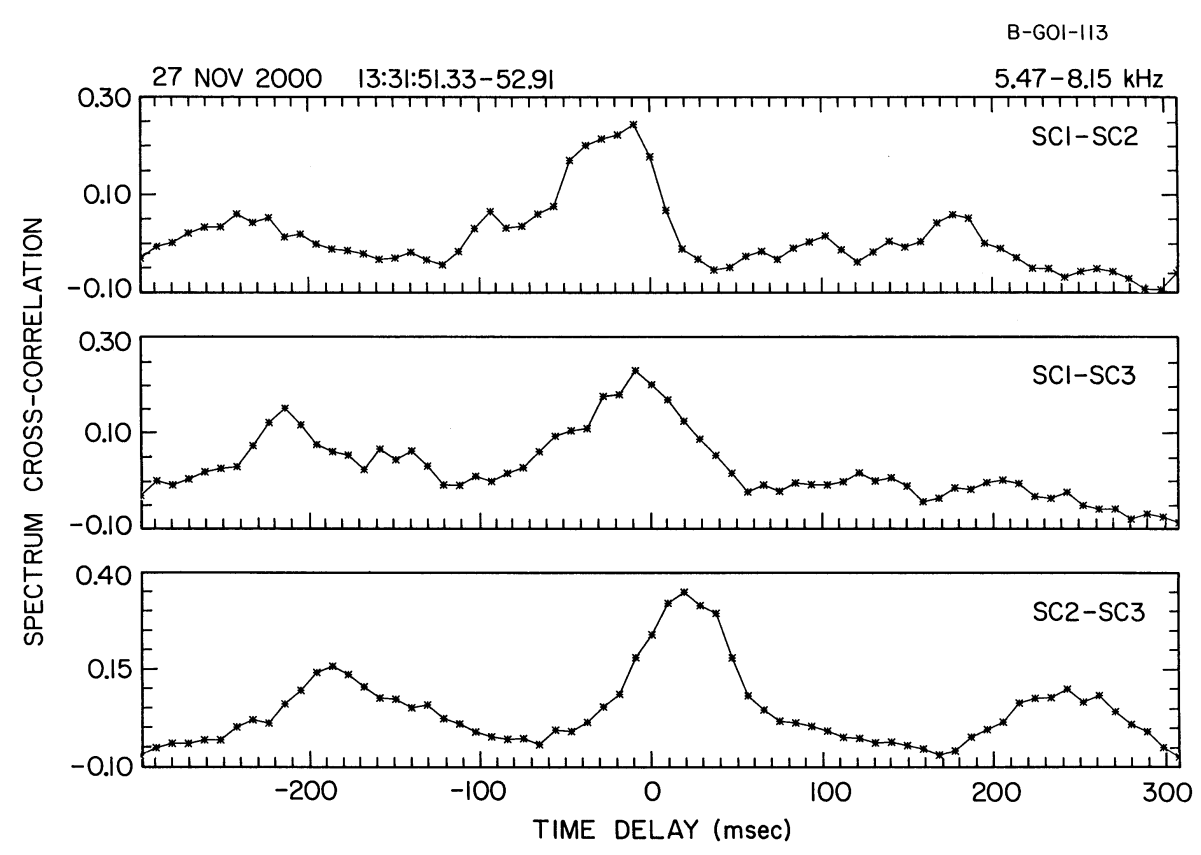

Fig. 10. The relative geometry of spacecraft SC1, SC2 and SC3 in magnetic field-aligned coordinates at 13:31 UT on 27 November 2000, using the Tsyganenko model for the Earth's magnetic field (Tsyganenko, 1989). At this time, the spacecraft were well north of the magnetic equator. Note that the relative separation in the $\Delta X-\Delta Y$ plane, transverse to the magnetic field, has increased compared to Figs. 8 and 9. This trend is caused by the downward tilt of the magnetic field in the magnetic meridian plane as the spacecraft move northward away from the magnetic equator.

Fig. 11. The cross-correlation between the three spacecraft as a function of time delay for a selected time interval from the spectrogram in Fig. 7. The frequency and time ranges over which the cross-correlation was performed were 5.47 to $8.15 \mathrm{kHz}$ and $13: 31: 51.33$ to 13:31:52.91 UT. A positive time delay indicates that the signal arrives first at the spacecraft listed first in the identifier for each panel (i.e. SC2 in the bottom panel, etc.).

received from SC3. Thus, for the spectrograms in Fig. 5, data are only available from SC1 and SC2. Nevertheless, these spectrograms are very interesting, since there is a very clear one-to-one correspondence between the chorus elements detected by the two spacecraft. Note that although the chorus elements look similar at the two spacecraft, the frequencies at $\mathrm{SC} 1$ are shifted upward by about $1 \mathrm{kHz}$ relative to $\mathrm{SC} 2$. Although it appears that the chorus elements arrive at $\mathrm{SC} 2$ before $\mathrm{SC} 1$, if one compares the arrival times at a fixed frequency, for example at $8.5 \mathrm{kHz}$ for the chorus element at 13:13:15.8 UT, one can clearly see that the signal arrives at $\mathrm{SC} 1$ before $\mathrm{SC} 2$. Referring to the $\Delta Y-\Delta Z$ panel of Fig. 8, one can see that the chorus wave packet is propagating southward, away from the magnetic equator, which is consistent with the Poynting flux measurements of LeDocq et al. (1998) who showed that the source of chorus emissions is located very close to the magnetic equator. From the transverse $\Delta X-\Delta Y$ plot in Fig. 8, one can see that SC1 and SC2 are very nearly on the same magnetic field line at this time. The separation distance perpendicular to the magnetic field is only about $200 \mathrm{~km}$. Since the whistler-mode energy propagates very nearly along the magnetic field lines, the small transverse separation relative to the magnetic field is most likely the reason that there is such a good one-to-one correspondence between the chorus elements at this time.

Over the next few minutes, as the spacecraft approach the magnetic equator, the clear one-to-one correlation evident at 13:13:15 UT in Fig. 5 gradually deteriorates. By 13:18:06 UT in Fig. 6, very close to the magnetic equator, a one-to-one correspondence between the chorus elements 


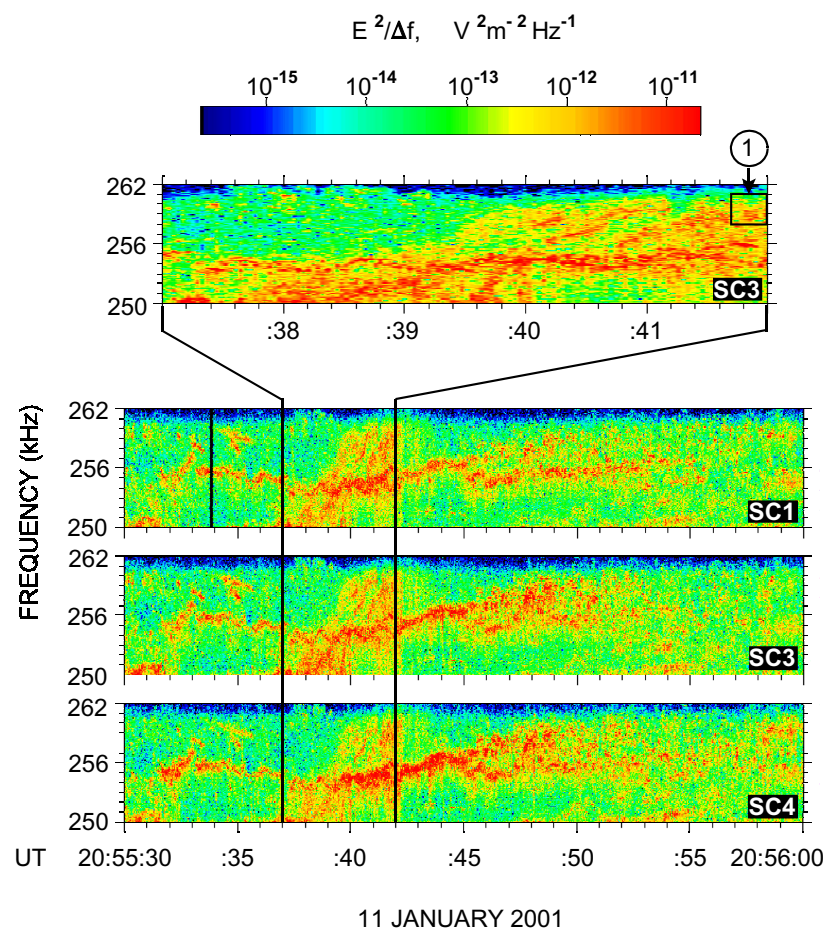

Fig. 12. AKR spectra recorded on spacecraft SC1, SC3, and SC4 on 11 January 2001 between 20:55:30 and 20:56:00 UT. The upper panel is an enlargement of the five-second time range 20:55:37 to 20:55:42. The box labeled " 1 " is the frequency-time segment used to calculate the cross-correlations shown in Fig. 14.

can no longer be discerned between any of the three spacecraft. Note that in the $\Delta X-\Delta Y$ plot of Fig. 9, the transverse distances perpendicular to the magnetic field are still quite small, less than $300 \mathrm{~km}$. The one-to-one correspondence continues to remain poor as the spacecraft proceed northward away from the magnetic equator. However, occasionally short periods exist where a clear one-to-one correspondence can be found. For example, in Fig. 7 from about 13:31:50 to 13:31:53 UT, a one-to-one correspondence can be seen between all three spacecraft, although the intensities are distinctly lower at SC1. The correspondence between the individual chorus elements is particularly good between SC2 and SC3. Note that there is no evidence of a frequency shift comparable to what was observed in Fig. 5. The differences in the arrival times are very small and cannot be easily measured by visually comparing the spectrograms. To analyze the difference in the arrival times, we have computed the cross-correlations between the spectrums as a function of time delay for frequencies from 5.47 to $8.15 \mathrm{kHz}$ and times from 13:31:51.33 to 13:31:52.91 UT. The results are shown in Fig. 11. As can be seen in the figure, the cross-correlations have peaks for negative time delays of about 10 milliseconds for the SC1-SC2 and SC1-SC3 cross-correlations, and a peak for a positive time delay of about 20 milliseconds for the SC2-SC3 cross-correlation. The negative time delay between $\mathrm{SC} 1$ and $\mathrm{SC} 2$ means that the signals arrived at SC2 before $\mathrm{SC} 1$, and the negative time delay between $\mathrm{SC} 1$ and

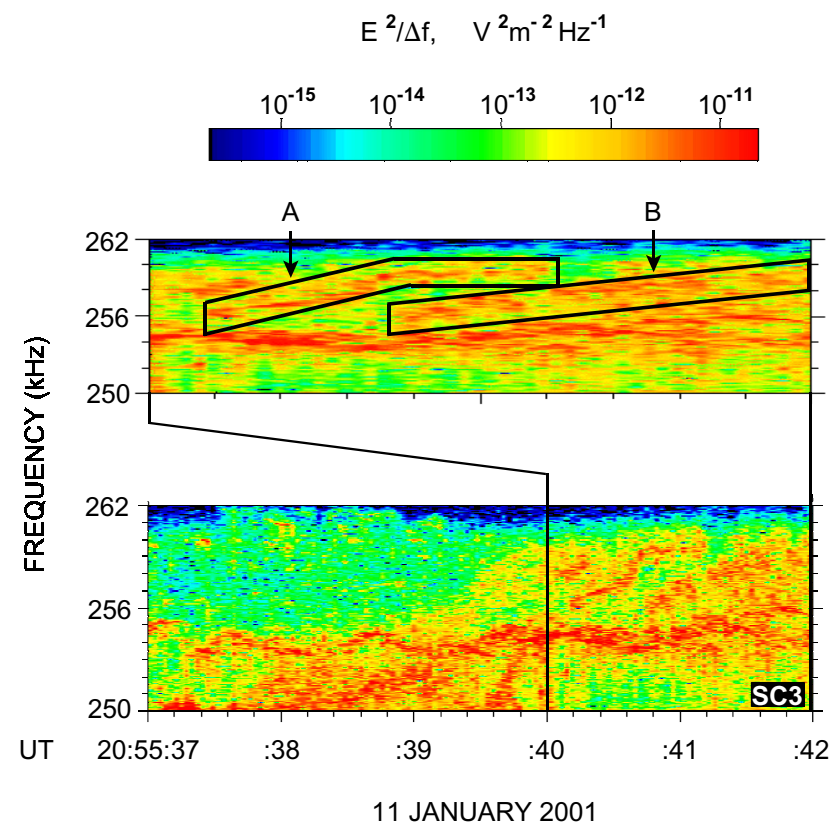

Fig. 13. (a) Two-second spectrum of AKR emission from SC3 on 11 January 2001 between 20:55:40 and 20:55:42 UT. Note the two narrowband, drifting AKR sources labeled A and B. Crosscorrelation analysis of both events were performed with similar measured delays. The cross-correlations shown in Fig. 14 were calculated using a 300-msec time window near the end of the source B track. (b) Five-second spectrum showing the nearby AKR activity at the time of events $\mathrm{A}$ and $\mathrm{B}$.

$\mathrm{SC} 3$ means that the signals arrived at SC3 before SC1. Similarly, the positive time delay between $\mathrm{SC} 2$ and $\mathrm{SC} 3$ means that the signals arrived at $\mathrm{SC} 2$ before $\mathrm{SC} 3$. Referring to the $\Delta Y-\Delta Z$ panel of Fig. 10, one can see that these time delays show that the chorus is propagating northward (toward positive $\Delta Z$ ). Since the spacecraft are now north of the magnetic equator, the direction of propagation indicates the chorus is propagating away from the magnetic equator, which is again consistent with the Poynting flux measurements of LeDocq et al. (1998). Note that the direction of energy propagation has reversed as the spacecraft crossed the magnetic equator. In addition, there is no evidence in Fig. 11 of chorus elements propagating toward the magnetic equator, which is again consistent with the result of LeDocq et al. (1998) who showed that there is little evidence of reflected waves returning along the same magnetic field lines. From the transverse $\Delta X-\Delta Y$ plot in Fig. 10, one can see that by 13:31 UT the separation distance perpendicular to the magnetic field has increased markedly, to a maximum of about $500 \mathrm{~km}$. The increased transverse separation is due to the downward tilt of the magnetic field as the spacecraft move northward away from the magnetic equator. After about this time, the one-toone correspondence between the chorus elements at the various spacecraft deteriorates markedly, as the transverse separation distance continues to increase. Thus, $500 \mathrm{~km}$ appears to be approximately the outer limit for the dimensions of the chorus wave packets transverse to the magnetic field. 


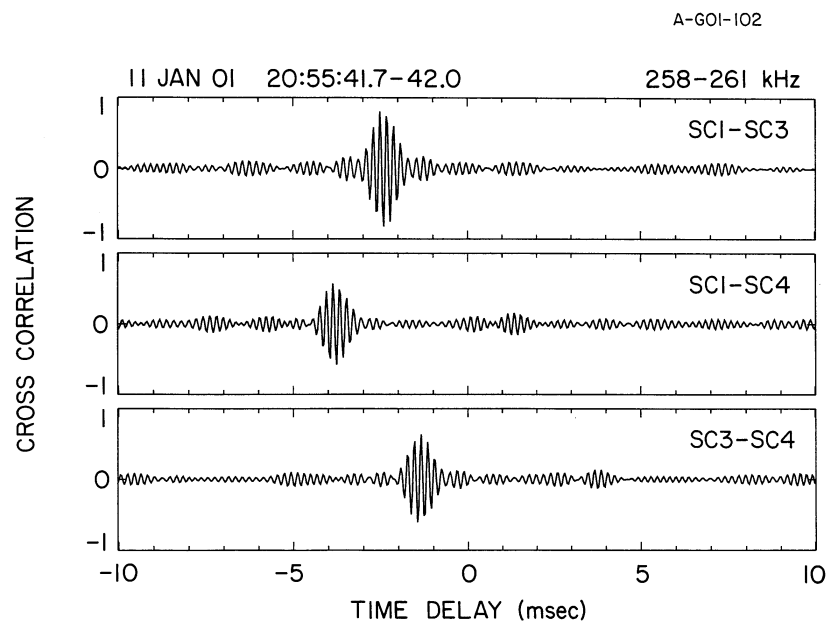

Fig. 14. Cross-correlation amplitude versus delay for AKR source on 11 January 2001 at 20:55:42 UT for baselines SC1-SC3, SC1$\mathrm{SC} 4$, and SC3-SC4. The observed delay maximums are used to determine source location by triangulation.

Prior to these observations, the typical size of a chorus wave packet was essentially unknown. The only known constraint was that it had to be substantially larger than the wavelength, i.e. greater than a few kilometers. These observations show that the largest transverse $\Delta X-\Delta Y$ distance, perpendicular to the magnetic field, at which an easily identifiable inter-spacecraft correlation can be identified is about $500 \mathrm{~km}$ (see Fig. 8). This distance is apparently near the outer limit for the transverse dimensions of the chorus wave packets, since a good one-to-one correspondence between the chorus elements was not always present at this separation distance. The direction of propagation observed during the 27 November pass also showed the expected reversal near the magnetic equator, with the chorus elements propagating away from the magnetic equator, and with little or no evidence of the wave reflecting back along the same magnetic field line. In the region within a few degrees of the magnetic equator, presumably in or near the source region, the situation was very confused, with very little evidence of a one-to-one correspondence between the chorus elements, even though the minimum transverse separation distance between the spacecraft was quite small, less than $300 \mathrm{~km}$. Finally, it is interesting to note the highly unusual frequency shift for the chorus element observed by SC1 and SC2 in Fig. 5. Clearly, the two spacecraft are detecting the same chorus wave packets, since they occur in a one-to-one correspondence and have very similar frequency-time shapes. However, the frequencies of the wave packets detected by SC1 are shifted upward relative to those detected by SC2. Such a frequency shift could either indicate (1) that the frequency of the packet has evolved in the process of propagating from SC1 to SC2, or (2) that the two spacecraft are sampling different regions of the same wave packet, possibly due to the frequency dependence of the ray paths from the source region to the spacecraft. Temporal evolution of the chorus el- ements is expected as the wave packet evolves in the source region (Helliwell, 1967). Ground-based observations of discrete chorus elements with rising and falling frequency-time signatures (Helliwell, 1965) indicate that frequency variations are inherent in the source mechanism. Whether the observed frequency shifts are an example of such a temporal evolution, or whether they can be explained by relatively simple propagation effects remains to be determined. In principal, these two possibilities could be resolved if simultaneous wave vector measurements had been available. Unfortunately, in this case, the frequencies of the chorus elements are above the 4-kHz upper frequency limit of the STAFF instrument, which can provide wave vector measurements (Cornilleau et al., 1997). Perhaps in the future, some chorus events can be found where such comparisons will be possible.

\section{Auroral Kilometric Radiation}

During the mid 1960s and early 1970s an intense radio emission was detected escaping from the Earth in the frequency range from about 50 to $500 \mathrm{kHz}$. The first observations of this radiation were by Benediktov et al. (1968) who detected a radio emission of unknown origin escaping from the Earth at frequencies of 725 and $1100 \mathrm{kHz}$ that was correlated with the $K p$ planetary magnetic disturbance index. Dunckel et al. (1970) also observed what they termed high-pass noise, primarily due to the low frequency part of the spectrum below about $100 \mathrm{kHz}$. A few years later, Gurnett (1974) showed that the radiation had its peak intensity in the frequency range from about 100 to $500 \mathrm{kHz}$, that it was very intense, approximately $10^{9}$ Watts of total radiated power, and that it was closely correlated with the occurrence of discrete auroral arcs. Since electromagnetic radiation in this frequency range has wavelengths in the kilometer range, and since there is a close association with the aurora, this radiation is now called auroral kilometric radiation, often abbreviated AKR. Subsequent studies have shown that AKR is generated along the nighttime auroral field lines (Kurth et al., 1975) at radial distances from about 2 to $4 R_{E}$ (Gallagher and Gurnett, 1979), and is primarily generated in the right-hand polarized mode (Shawhan and Gurnett, 1982) at frequencies very close to the electron cyclotron frequency (Huff et al., 1988). Gurnett et al. (1979) also showed that the AKR spectrum is highly structured and consists of many narrowband emissions with rapidly varying center frequencies. Individual elements of the spectrum can have bandwidths as narrow as $100 \mathrm{~Hz}$ and time scales of a fraction of one second. Based on these results, it is now believed that auroral kilometric radiation is generated by a mechanism known as the cyclotron maser (Wu and Lee, 1979). In this mechanism, electrons are quasitrapped by the magnetic mirror force and the electric field (Louarn et al., 1990; Roux et al., 1993; Ergun et al., 1998; Delory et al., 1998). The cyclotron maser mechanism is also believed to be responsible for the intense radio emissions that have been observed from Jupiter, Saturn, Uranus, and Nep- 
tune. For a recent review of planetary radio emissions, see Zarka (1998).

One of the primary objectives of the Cluster WBD investigation is to use the unique four-spacecraft configuration of Cluster to conduct very-long-baseline interferometry (VLBI) investigations of AKR. VLBI measurements can provide much better angular resolution measurements of the AKR source than can be achieved with any other technique, and may be able to give highly detailed measurements of source sizes and motions (Mutel et al., 2000). Since Cluster can view the entire auroral oval when the spacecraft are at high altitudes over the polar regions, VLBI measurements have the potential of giving a global image of the AKR source distribution that is not possible with any other technique. The possibility of using VLBI techniques for the study of AKR has already been demonstrated by Baumback et al. (1986) who showed, by using measurements from the ISEE 1 and 2 spacecraft, that AKR interference fringes could be obtained for baseline separations as large as $3800 \mathrm{~km}$. These measurements showed that the source of the individual elements of the AKR spectrum was as small $20 \mathrm{~km}$. Since only two spacecraft were available no information could be obtained on source positions or motions using the ISEE data.

Figure 12 illustrates sample spectrums detected on spacecraft SC1, SC3 and SC4 in a 30-second interval starting at 20:55:30 UT on 11 January 2001. The times in these spectrograms are ground received times. These data were obtained in the frequency conversion mode over the nighttime polar region at a geocentric radial distance of $5.9 R_{E}$. The frequency range was from 250.0 to $259.5 \mathrm{kHz}$, roughly in the middle of the most intense part of the AKR emission band. The spectrums show several individual short duration AKR emission features, with the typical drifting finestructure characteristic of AKR. The spectral features are clearly highly correlated between the three spacecraft.

In "normal" VLBI observations of radio-astronomical sources (Thompson et al., 1986), the emission frequency is stable and typically has a much broader bandwidth than the bandwidth of the receiving system. Hence, the width of the correlation delay function is given by the reciprocal of the receiver bandwidth, typically under one microsecond. However, the AKR emission has a very narrow bandwidth (typically $\Delta f<1 \mathrm{kHz}$ ) and varies rapidly both in time and frequency (see Fig. 12). This means that the uncertainty in the measured time delay is determined by the instantaneous bandwidth of the AKR signal, which varies from event to event. In order to determine the source angular size and direction of arrival, we cross-correlated the waveforms from pairs of spacecraft. The amplitude of the peak correlation is a measure of the angular size, while the differential time delays between pairs of spacecraft can be used to find the source position by "triangulation". In order to compute the cross-correlation versus time delay for an individual AKR feature, we must first isolate the feature both spectrally and temporally. To do this, we applied digital filters to the raw waveform. The drifting AKR features labeled A and B in Fig. 13 were selected by applying a moving frequency band-

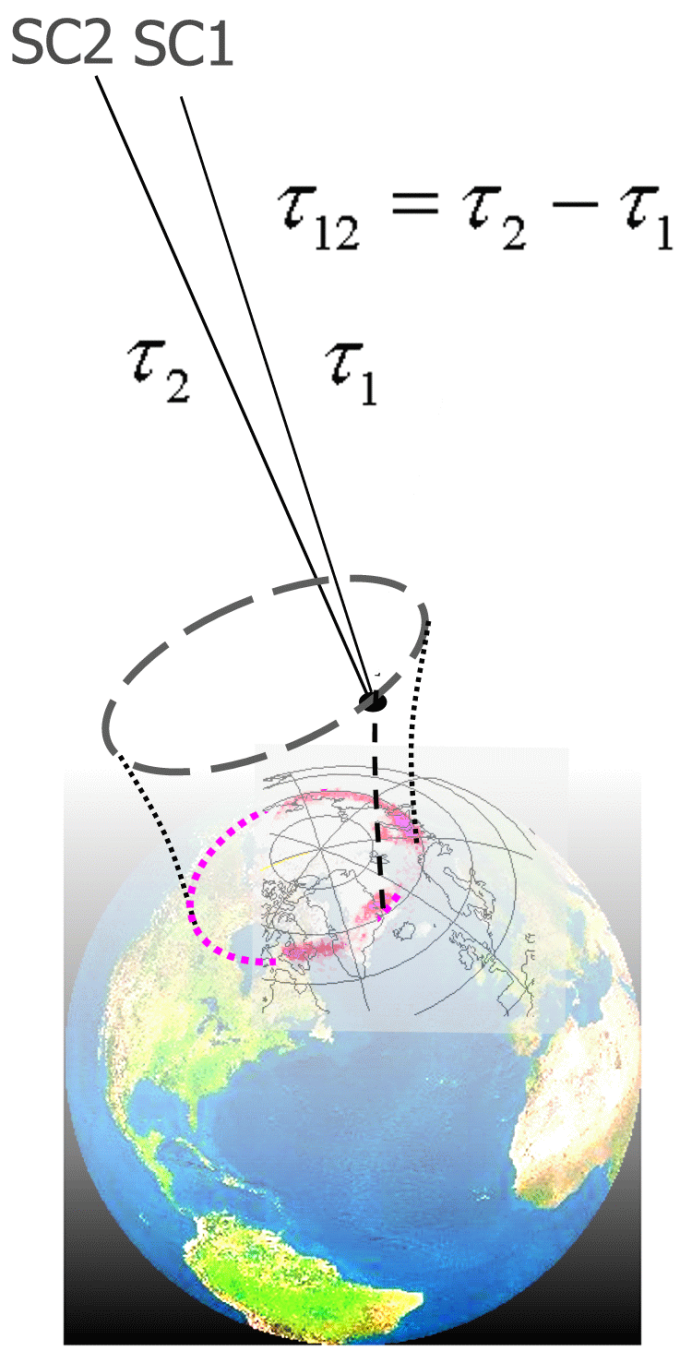

Fig. 15. Geometry of AKR source location scheme. The source is constrained to locations in a ring $1.8 R_{E}$ from the Earth's center and projected outward along magnetic field lines traversing the auroral oval imaged at the time of the observed AKR emission.

pass filter (shown as the black boxes), and then computing a cross-correlation every $300 \mathrm{msec}$.

The resulting cross-correlation for the AKR source labeled B on 11 January 2001 at 20:55:42 UT is shown in Fig. 14. The box marked "1" in Fig. 12 shows the actual $300 \mathrm{msec}$ sample used to compute the cross-correlation in Fig. 14. The maximum cross-correlation amplitude varies from 0.6 to 0.9 . Since the correlation amplitude does not appear to depend on the sky-projected baseline length (e.g. $60 \mathrm{~km}$ for SC1$\mathrm{SC} 3$ vs. $1064 \mathrm{~km}$ for $\mathrm{SC} 1-\mathrm{SC} 4)$, it suggests that the source is unresolved, and the observed reduction from perfect correlation is caused by the addition of uncorrelated background noise in the bandpass signal. We tentatively conclude that the Gaussian-equivalent source angular size is less than $4 \mathrm{arcmin}$ (42 $\mathrm{km}$ at a distance $\sim 5 R_{E}$ ).

The measurement of source position is determined by triangulation using the observed baseline differential delays. 


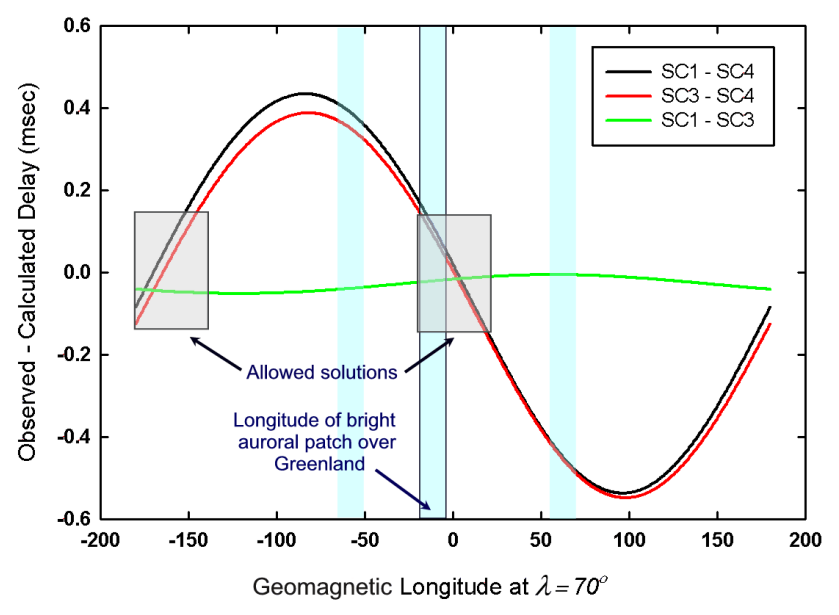

Fig. 16. The delay difference (observed minus calculated) for baselines SC1-SC3, SC1-SC4, and SC3-SC4 versus ring longitude for the AKR emission event of 11 January 2001 at 20:55:42 UT. The light blue strips are longitudes of auroral bright spots as seen on the Polar UVI image; the boxes are the allowed regions constrained by the observed delays and their uncertainties. There are two allowed longitude ranges, but only one (near $-20^{\circ}$ longitude) is along a magnetic field line connected to a bright auroral spot. The AKR source is believed to be located within this region.

For a baseline connecting spacecraft a and $b$, the observed delay can be written

$\tau_{a b}=\frac{1}{c} \cdot\left(\left|s_{b}-r\right|+\left|t_{b}-s_{b}\right|-\left|s_{a}-r\right|-\left|t_{a}-s_{a}\right|(1)\right.$

where $s$ are (geocentric) position vectors to spacecraft (a) and (b), $\boldsymbol{t}$ are position vectors to the downlink telescopes, and $\boldsymbol{r}$ is the source position vector. We have assumed that there are negligible clock synchronization errors among the downlink telescopes and that the differences in instrumental delays are also negligible. For observations using $\mathrm{n}$ spacecraft, there are $n(n-1) / 2$ independent baselines with only three unknowns, namely the Cartesian components of the source position $\boldsymbol{r}$. Therefore, in principle, a source position can be uniquely determined for observations using three or more spacecraft. In practice, this is only true if the path length difference between the arrival of the signal at either end of a given baseline satisfies of $\Delta \ell>c \delta \tau$, where $\delta \tau$ is the delay uncertainty in the correlation peak. Inspection of Fig. 14 shows that delay maximums can be determined to an uncertainty $\delta \tau \sim 150 \mu \mathrm{sec}$, so that the path length differences on each baseline must be larger than $\Delta \ell \sim 45 \mathrm{~km}$. This was only true for two of the three baselines for the event of 11 January 2001, so we imposed a constrained source position locus as described below. As the Cluster configuration expands to longer baselines, it will be much easier to solve for source position without ancillary assumptions.

In order to solve for the AKR source position for the 11 January 2001 event, we made two a priori assumptions. The first was that the source was radiating at the local electron cyclotron frequency (Gurnett, 1974; Huff et al., 1988) where

$f_{c}(M H z)=2.8 B_{\text {gauss }}$
For the examples discussed below, the observed frequency varied from $250-262 \mathrm{kHz}$. Hence, the ambient magnetic field in the source region is $B \sim 0.09$ gauss. Second, we assumed that the AKR emission arises from magnetic field lines connecting regions of auroral activity (Huff et al., 1988). For a dipole field approximation, the magnetic field strength at radial distance $R$ and magnetic latitude $\lambda_{m}$ is

$B\left(R, \lambda_{m}\right)=\frac{0.3 \text { gauss }}{R^{3}} \cdot\left(3 \sin ^{2} \lambda_{m}+1\right)^{1 / 2}$

In order to determine the distribution of auroral emissions at the time of observation, we downloaded Polar UVI images (http://uvisun.msfc.nasa.gov) of the northern hemisphere (kindly provided by G. Parks and colleagues). By knowing the geomagnetic latitude of the auroral oval at the time of the observations and the ambient magnetic field of the AKR source, we can solve for the radial distance of the AKR source. For example, for the event analyzed in this paper (epoch 2001, 11 January 2001 at 20:55:40 UT), the auroral oval was at geomagnetic latitude $\lambda_{m} \sim+70^{\circ}$, implying a geocentric radial distance $R \sim 11800 \mathrm{~km}\left(1.8 R_{E}\right)$. Hence, the locus of possible AKR source locations is a circular annulus at a geocentric distance $R=1.8 R_{E}$ projected along a cone with vertex at the Earth's center and passing through the auroral oval (see Fig. 15).

In order to determine the source location in this oval for a given AKR event, we calculated the expected differential delays on all available baselines for sources located everywhere on the source annulus and compared them with the observed delays. An example is shown in Fig. 16, where we have plotted the difference between the observed and expected delay for each of three baselines (SC1-SC3, SC1-SC4, and SC3$\mathrm{SC} 4)$ as a function of geomagnetic longitude. Also shown as shaded vertical regions are the geomagnetic longitudes of the three bright points evident in the UVI auroral image. The delay differences are nearly zero for geomagnetic longitude ranges $-180^{\circ} \pm 20^{\circ}$ and $0^{\circ} \pm 20^{\circ}$. The bright auroral patch marked with an arrow in Fig. 16 encompasses the second range and is likely to be the origin of the AKR emission.

Note that for the Cluster configuration during this observation, the baseline $\mathrm{SC} 1-\mathrm{SC} 3$ was very insensitive to the transverse source position shift, since its projection in the sky plane was very small $(60 \mathrm{~km})$, compared with baselines SC1SC4 and SC3-SC4 (814 and $867 \mathrm{~km}$, respectively). Also note that we have not (yet) corrected for refractive ray bending or the deviation of magnetic field lines from the radial direction in this preliminary calculation. (For the event analyzed, the Cluster spacecraft were within $8^{\circ}$ of the magnetic pole at a distance of $5.9 R_{E}$, so that the refractive effects are small.) Finally, we are investigating more sophisticated algorithms for determining delay centroids (e.g. wavelets) to reduce the cross-correlation delay uncertainty. All of these issues will be incorporated in a more complete analysis in a future publication. Nevertheless, we are confident that the position determined is accurate to $\pm 20^{\circ}$ in geomagnetic longitude and provides the first direct confirmation that AKR sources are located above bright points on the auroral oval. 
We are now analyzing the position of several other AKR bursts at several epochs. The delay-locus technique works optimally for Cluster configurations with large projected baselines in the sky plane. It would also benefit enormously from real time downlinks from all four spacecraft, since the number of independent differential delays is doubled (6 vs. 3). As the Cluster configuration expands and baselines approach ten times the present configuration, we anticipate determining AKR source positions with an uncertainty under one hundred $\mathrm{km}$.

\section{Conclusions}

These results provide just an initial sample of the types of studies that are possible with the Cluster wideband plasma wave investigation. At present, due to the limited amount of data that have been collected via the NASA Deep Space Network, the range of phenomena that can be investigated is quite limited. However, as time evolves, it will be possible to obtain high-resolution waveform measurements of plasma waves from all of the important regions of the magnetosphere. These will include (1) further measurements of whistlers and whistler-mode chorus emissions near the magnetic equator in the inner region of the magnetosphere, (2) measurements of auroral hiss and other auroral plasma waves along the auroral field lines, (3) measurements of various types of plasma waves in the polar cusp, magnetosheath, and plasma sheet, and (4) very-long-baseline studies of various types of magnetospheric radio emissions, such as auroral kilometric radiation and terrestrial continuum radiation.

Acknowledgements. This project was supported by NASA through contract NAS5-30730 and grant NAG5-9974 from Goddard Space Flight Center. The authors thank Cindee Abramo, Romy Paredes, Tony Horton, and Steve Waldherr of JPL/DSN; and Ieuan Willis of the University of Sheffield and Mike Hapgood of Rutherford Appleton Laboratory for their help in scheduling and commanding the wideband operations. We acknowledge the efforts of Robert Brechwald, Julie Dowell, Jon Drieling, and Joe Groene of the University of Iowa for their help in the ground software development. We also thank Prof. George Parks for providing the UV image of the aurora, and Prof. A. Balogh, Principal Investigator of the magnetic field investigation, for providing the magnetic field data.

The Editor in Chief thanks two referees for their help in evaluating this paper.

\section{References}

Abel, B. and Thorne, R. M.: Electron scattering loss in the Earth's inner magnetosphere. 1. Dominant physical processes, J. Geophys. Res., 103, 2385-2396, 1998.

Balogh, A., Dunlop, M. W., Cowley, S. W. H., Southwood, D. J., Thomlinson, J. G., Glassmeier, K. H., Musmann, G., Lühr, H., Buchert, S., Acuña, M. H., Fairfield, D. H., Slavin, J. A., Riedler, W., Schwingenschuh, K., and Kivelson, M. G.: The Cluster magnetic field investigation, Space Sci. Rev., 79, 65-91, 1997.

Barkhausen, H.: Zwei mit Hilfe der neuen Verstärker entdeckten Erscheinungen, Physik. Zeitschr., 20, 401-403, 1919.
Baumback, M. M., Gurnett, D. A., Calvert, W., and Shawhan, S. D.: Satellite interferometric measurements of auroral kilometric radiation, Geophys. Res. Lett., 13, 1105-1108, 1986.

Benediktov, E. A., Getmantsev, G. G., Mityakov, N. A., Rapoport, V. O., and Tarasov, A. F.: Relation between geomagnetic activity and the sporadic radio emission recorded by the elektron satellites, Kosmicheskie Issledovaniya, 6, 946-949, 1968.

Brice, N. M.: Traveling wave amplification of whistlers, J. Geophys. Res., 65, 3830-3842, 1962.

Brice, N.: Fundamentals of very low frequency emission generation mechanisms, J. Geophys. Res., 69, 4515-4522, 1964.

Burgess, W. C. and Inan, U. S.: The role of ducted whistlers in the precipitation loss and equilibrium flux of radiation belt electrons, J. Geophys. Res., 98, 15, 643-665, 1993.

Burtis, W. J. and Helliwell, R. A.: Banded chorus - a new type of VLF radiation observed in the magnetosphere by OGO 1 and OGO 3, J. Geophys. Res., 74, 3002-3010, 1969.

Burtis, W. J. and Helliwell, R. A.: Magnetospheric chorus: Amplitude and growth rate, J. Geophys. Res., 80, 3265-3270, 1975.

Burton, R. K.: Critical electron pitch angle anisotropy necessary for chorus generation, J. Geophys. Res., 81, 4779-4781, 1976.

Burton, R. K. and Holzer, R. F.: The origin and propagation of chorus in the outer magnetosphere, J. Geophys. Res., 79, 10141023, 1974.

Carpenter, D. L. and Anderson, R. R.: An ISEE/whistler model of equatorial electron density in the magnetosphere, J. Geophys. Res., 97, 1097-1108, 1992.

Carpenter, D. L. and Smith, R. L.: Whistler measurements of electron density in the magnetosphere, Rev. Geophys., 2, 415-441, 1964.

Cornilleau-Wehrlin, N., Chauveau, P., Louis, S., Meyer, A., Nappa, J. M., Perraut, S., Rezeau, L., Robert, P., Roux, A., De Villedary, C., De Conchy, Y., Friel, L., Harvey, C. C., Hubert, D., Lacombe, C., Manning, R., Wouters, F., Lefeuvre, F., Parrot, M., Pincon, J. L., Poirier, B., Kofman, W., and Louarn, P.: The Cluster spatio-temporal analysis of field fluctuations (STAFF) experiment, Space Sci. Rev., 79, 107-136, 1997.

Delory, G. T., Ergun, R. E., Carlson, C. W., Muschietti, L., Chaston, C. C., Peria, W., McFadden, J. P., and Strangeway, R.: FAST observations of electron distributions within AKR source regions, Geophys. Res. Lett., 25, 2069-2072, 1998.

Draganov, A. B., Inan, U. S., Sonwalker, V. S., and Bell, T. F.: Magnetospherically reflected whistlers as a source of plasmaspheric hiss, Geophys. Res. Lett., 19, 233-236, 1992.

Dunckel, N., Ficklin, B., Rorden, L., and Helliwell, R. A.: Lowfrequency noise observed in the distant magnetosphere with OGO 1, J. Geophys. Res., 75, 1854-1862, 1970.

Eckersley, T. L.: Musical atmospherics, Nature, 135, 104-105, 1935.

Edgar, B. C.: The upper and lower cutoffs of magnetospherically reflected whistlers, J. Geophys. Res., 81, 205, 1976.

Ergun, R. E., Carlson, C. W., McFadden, J. P., Mozer, F. S., Delory, G. T., Peria, W., Chaston, C. C., Temerin, M., Elphic, R., Strangeway, R., Pfaff, R., Cattell, C. A., Klumpar, D., Shelly, E., Peterson, W., Möbius, E., and Kistler, L.: FAST satellite wave observations in the AKR source region, Geophys. Res. Lett., 25, 2061-2064, 1998.

Escoubet, C. P., Schmidt R., and Goldstein, M. L.: Cluster - science and mission overview, Space Sci. Rev., 79, 11-32, 1997.

Gallagher, D. L. and Gurnett, D. A.: Auroral kilometric radiation: Time-averaged source location, J. Geophys. Res., 84, 65016509, 1979. 
Gurnett, D. A.: The Earth as a radio source: Terrestrial kilometric radiation, J. Geophys. Res., 79, 4227-4238, 1974.

Gurnett, D. A. and Inan, U. S.: Plasma wave observations with the Dynamics Explorer 1 spacecraft, Rev. Geophys., 26, 285-316, 1988.

Gurnett, D. A. and O'Brien, B. J.: High-latitude geophysical studies with satellite Injun 3, 5. Very-low-frequency electromagnetic radiation, J. Geophys. Res., 69, 65-89, 1964.

Gurnett, D. A., Anderson, R. R., Scarf, F. L., Fredricks, R. W., and Smith, E. J.: Initial results from the ISEE 1 and 2 plasma wave investigation, Space Sci. Rev., 23, 103-122, 1979.

Gurnett, D. A., Huff, R. L., and Kirchner, D. L.: The wide-band plasma wave investigation, Space Sci. Rev., 79, 195-208, 1997.

Helliwell, R. A.: Whistlers and Related Ionospheric Phenomena, Stanford University Press, Stanford, CA, 1965.

Helliwell, R. A.: A theory of discrete VLF emissions from the magnetosphere, J. Geophys. Res., 72, 4773-4790, 1967.

Helliwell, R. A.: Low-frequency waves in the magnetosphere, Rev. Geophys., 7, 281-303, 1969.

Huff, R. L., Calvert, W., Craven, J. D., Frank, L. A., and Gurnett, D. A.: Mapping of auroral kilometric radiation sources to the aurora, J. Geophys, Res., 93, 11 445-11 454, 1988.

Johnson, M. P., Inan, U. S., and Lauben, D. S.: Subionospheric VLF signatures of oblique (nonducted) whistler-induced precipitation, Geophys. Res. Lett., 26, 3569-3572, 1999.

Kennel, C. F. and Petschek, H. E.: Limit on stably trapped particle fluxes, J. Geophys. Res., 71, 1-28, 1966.

Kurth, W. S., Baumback, M. M., and Gurnett, D. A.: Directionfinding measurements of auroral kilometric radiation, J. Geophys. Res., 80, 2764-2770, 1975.

Lauben, D. S., Inan, U. S., Bell, T. F., Kirchner, D. L., Hospodarsky, G. B., and Pickett, J. S.: VLF chorus emissions observed by Polar during the 10 January 1997 magnetic cloud, Geophys. Res. Lett., 25, 2995-2998, 1998.

LeDocq, M. J., Gurnett, D. A., and Hospodarsky, G. B.: Chorus source locations from VLF Poynting flux measurements with the Polar spacecraft, Geophys. Res. Lett, 25, 4063-4066, 1998.

Louarn, P., Roux, A., De Feraudy, H., Le Queau, D., André, M., and Matson, L.: Trapped electrons as free energy source for the auroral kilometric radiation, J. Geophys. Res., 95, 5983-5995, 1990.

McIlwain, C. E.: Coordinates for mapping the distribution of magnetically trapped particles, J. Geophys. Res., 66, 3681-3691, 1961.

Mutel, R. L., Gurnett, D. A., and Huff, R. L.: VLBI studies of auroral kilometric radiation and solar type III bursts using the wideband data plasma wave instrument, Proc. Cluster II Workshop on Multiscale/Multipoint Plasma Measurements, London, 22-24 Sept. 1999, ESA SP-449, February 2000.

Nunn, D., Omura, Y., Matsumoto, H., Nagano, I., and Yagitani, S.: The numerical simulation of VLF chorus and discrete emissions observed on the Geotail satellite using a Vlasov code, J. Geophys. Res., 102, 27 083-27 097, 1997.

Pedersen, A., Cornilleau-Wehrlin, N., De La Porte, B., Roux, A., Bouabdellah, A., Décréau, P. M. E., Lefeuvre, F., Sene, F. X., Gurnett, D., Huff, R., Gustafsson, G., Holmgren, G., Woolliscroft, L., Alleyne, H. St. C., Thompson, J. A., and Davies, P. H. N.: The wave experiment consortium (WEC), Space Sci. Rev., 79, 93-105, 1997.

Roux, A., Hilgers, A., De Feraudy, H., Le Queau, D., Louarn, P., Perraut, S., Bahnsen, A., Jespersen, M., Ungstrup, E., and André, M.: Auroral kilometric radiation sources: In situ and remote observations from Viking, J. Geophys. Res., 98, 11, 657-670, 1993.

Salvati, M. A., Inan, U. S., Rosenberg, T. J., and Weatherwax, A. T.: Solar wind control of polar chorus, Geophys. Res. Lett., 27, 649-652, 2000.

Sazhin, S. S. and Hayakawa, M.: Magnetospheric chorus emissions: A review, Planet. Space Sci., 40, 681-697, 1992.

Shawhan, S. D. and Gurnett, D. A.: Polarization measurements of auroral kilometric radiation by Dynamics Explorer-1, Geophys. Res. Lett., 9, 913-916, 1982.

Smith, R. L. and Angerami, J. J.: Magnetospheric properties deduced from OGO 1 observations of ducted and non-ducted whistlers, J. Geophys. Res., 73, 1-20, 1968.

Smith, R. L., Helliwell, R. A., and Yabroff, I. W.: A theory of trapping of whistlers in field-aligned columns of enhanced ionization, J. Geophys. Res., 65, 815-823, 1960.

Sonwalker, V. S. and Inan, U. S.: Lightning as an embryonic source of VLF hiss, J. Geophys. Res., 94, 6986-6994, 1989.

Stix, T. H.: The Theory of Plasma Waves, McGraw-Hill, New York, 1962.

Storey, L. R. O.: An investigation of whistling atmospherics, Phil. Trans. Roy. Soc. London, A246, 113-141, 1953.

Thompson, R., Moran, J. M., and Swenson, Jr., G. W.: Interferometry and Synthesis in Radio Astronomy, John Wiley and Sons, New York, 1986.

Tsyganenko, N. A.: A magnetic field model with a warped tail current sheet, Planet. Space Sci., 37, 5-20, 1989.

Wu., C. S. and Lee, L. C.: A theory of the terrestrial kilometric radiation, Astrophy. J., 230, 621-626, 1979.

Yabroff, I.: Computation of whistler ray paths, J. Res. Nat. Bureau of Standards-D. Radio Propagation, 65D(5), 485-505, 1961.

Zarka, P.: Auroral radio emissions at the outer planets: Observations and theories, J. Geophys. Res., 103, 20 159-20 194, 1998. 